\section{Mejora de Procesos utilizando Respuestas Cualitativas mediante el uso de Redes NeuRONAles}

Recepción: Febrero de 2005 / Aceptación: Junio 2005
(1) Juan Cevallos Ampuero

(2) Roberto Eyzaguirre Tejada

\section{RESUMEN}

La aplicación de Redes Neuronales en problemas de optimización de parámetros para mejora de procesos en los que las respuestas de calidad son cualitativas, es el tema de esta investigación. Se fundamenta una propuesta de metodología para mejora de calidad de procesos usando redes neuronales y una aplicación de estas en la solución de problemas en los que las respuestas de calidad son cualitativas.

Palabras Clave: Mejora de procesos. Respuestas cualitativas. Redes neuronales.

Process Improvement using Qualitative Answers by means of Neuronal Networks ABSTRACT

The article deals with the application of Neura Networks in parameters optimization problems for the improvement of processes in which the answers of quality are qualitative. We propose a methodology to improve the quality of processes using neural networks and present an application of neural networks in the solution of problems in which the answers of quality are qualitative.

Key words: Improvement of processes. Qualitative answers. Neuronal network.



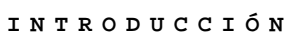

En la actualidad los mayores esfuerzos por mejorar la calidad se realizan fuera de la línea de producción. Es decir están referidos al diseño. Ello es fundamental para un buen control de calidad en la línea de producción. Las características de calidad del producto final dependen de los factores controlables y no controlables (factores ruido). Por ello se buscan los parámetros óptimos de dichos factores para los procesos y así lograr mejoras importantes de la calidad.

Existen diversos trabajos con relación a procesos que se pueden medir de manera cuantitativa, pero poco sobre como trabajar con mediciones cualitativas. Sin embargo, en diversos casos la respuesta de calidad de interés puede ser cualitativa. Por ejemplo, la calidad de la superficie de un caramelo podría ser: no defectuosa, ligeramente defectuosa y fuertemente defectuosa.

De la revisión de la literatura se aprecian dos limitaciones al trabajo con valores cualitativos: a) Sólo los niveles de calidad óptima pueden ser obtenidos aún trabajando con factores controlables que usen valores continuos. (por ejemplo usando -1,0,1 para indicar niveles bajo, medio y alto) b) la profundidad del análisis estadístico dificulta su uso para técnicos con poco conocimiento de estadística.

Las redes neuronales están siendo usadas en una amplia gama de aplicaciones, que van desde clasificación y reconocimiento de patrones hasta optimización y control. Las redes neuronales se han empleado exitosamente para modelar sistemas con relaciones lineales y no lineales que están detrás de arquitecturas complejas. El uso de redes neuronales para modelar las relaciones entre las entradas (factores controlables) y salidas (valores objetivo) de experimentos es un enfoque más simple que la mayoría de métodos estadísticos tradicionales. El objeto de este trabajo es desarrollar una propuesta de optimización de parámetros basado en redes neuronales cuando las respuestas de calidad son cualitativas. Se construyen dos redes neuronales: una para obtener los valores óptimos de los factores controlables y la otra para predecir los resultados de las respuestas de calidad, que involucran valores cualitativos.

METODOS TAGUCHI Y OTROS RELACIONADOS

El Dr. Genichi Taguchi (10), desarrolló el Análisis Acumulativo AA para resolver efectivamente los problemas con respuestas cualitativas. Este método consiste básicamente de cuatro pasos:1) definir las correspondientes categorías acumulativas y los datos a obtener 2) cálculo 
inicial de las probabilidades acumuladas de cada categoría y determinar los efectos de los niveles de factor sobre las probabilidades acumuladas 3 ) plotear las probabilidades acumuladas y determinar la combinación óptima de valores y niveles, y 4) predicción de las probabilidades acumuladas de cada categoría bajo condiciones óptimas. Siguiendo, en parte lo propuesto por Taguchi, Nair (9) presentó 2 esquemas de puntuación para identificar separadamente la dispersión y ubicación de los efectos de cada factor controlable. El cuadrado medio es recomendado como evidencia de efectos importantes. Las condiciones de solución óptima de ambos efectos puede ser obtenida de acuerdo a la contribución del efecto dispersión y efecto ubicación de cada factor controlable.

La combinación óptima de niveles de factores controlables es obtenida estableciendo la relación entre el efecto dispersión y el efecto localización. Este enfoque es complejo y requiere del uso de la computadora. Los ajustes finales están supeditados a la experiencia de los ingenieros que usen su criterio subjetivo para comprimir los efectos dispersión y ubicación. En un trabajo más reciente Jear (6) utiliza un esquema de puntajes con ponderación de probabilidades para mejorar la propuesta de Nair (9).

Esta propuesta combina los efectos de dispersión y ubicación en una simple desviación del cuadrado medio. De acuerdo a la definición del tipo de datos, la característica apropiada es empleada y, se puede obtener el valor esperado de la desviación cuadrática media. La combinación de niveles de factores controlables es obtenida seleccionando la mínima desviación del cuadrado medio. Aunque los tres métodos de Taguchi (10), Nair (9) y Jear (6) pueden obtener optimización de parámetros, ellos sólo pueden determinar niveles de categoría óptimos. Ellos no pueden determinar óptimos continuos para valores establecidos de factores controlables. Recientemente, Hsieh y Tong (4), en el 2000 desarrollan una metodología sobre optimización de parámetros de respuestas de calidad con categorías de orden linguístico y para ello utilizan redes neuronales, con una metodología de 4 pasos y con 9 subpasos, lo cual constituye un aporte importante.

\section{REDES NEURONALES}

Las redes neuronales consisten de un número de neuronas artificiales altamente interconectadas. Una red neuronal puede modelar relaciones no lineales entre entradas y salidas de un sistema. La estructura oculta de las relaciones no lineales puede ser

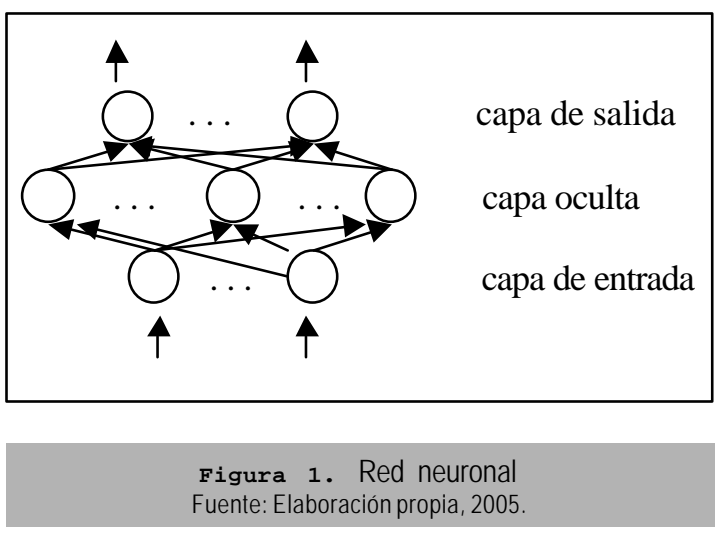

aprendida mediante el paso de pares de entrenamiento a través de la red. Existen diversos modelos de redes neuronales de aprendizaje supervisado, entre los que se incluye el perceptrón, la red neuronal con backpropagation, la red neuronal con organización mediante vector de aprendizaje LVQ y, la red con contador de propagación. El modelo de red con algoritmo backpropagation es el frecuentemente usado.

La red con el algoritmo backpropagation consiste en una capa de entrada, una de salida y una o más ocultas (ver Figura 1).

Para desarrollar una red conbackpropagation, primero hay que obtener los datos de entrenamiento y prueba. El conjunto de datos consiste: de valores de entrada y de salida del sistema. El algoritmo de aprendizaje del backpropagation emplea un gradiente heurístico que permite que una red se auto organice de tal manera que pueda mejorar su desempeño todo el tiempo.

Este paso a través de la red backpropagation empieza cuando la capa de entrada recibe los datos de entrada y pasan a través de la capa oculta, hasta la capa de salida. Cada neurona calcula una función de activación primero, considerando los valores de las entradas. Ello es usado luego como una función de activación en cada neurona para determinar el nivel de actividad de la neurona del proceso. En el entrenamiento de este tipo de red, un patrón de entrada es presentado y la red ajusta el conjunto de pesos en todas las conexiones; de tal forma que la salida deseada es obtenida en la neurona de salida. Alcanzado el ajuste, la red aprendió la asociación, y se puede dar valores de entrada para predecir los de salida.

El algoritmo de entrenamiento para la red usando la técnica de gradiente descendente es: 
Inicializar los pasos entre las capas, $\Delta W_{k j} y \Delta W_{j i}$; ver

Figura 1.

Para $\mathrm{m}=1$ hasta capa de salida de número de iteracción o criterio de error hacer

Decrecer gradualmente la tasa de ajuste para $\Delta W_{k j} y \Delta W_{j i}$;

Para $n=1$ hasta entrenar tamaño de muestras hacer

Calcular la salida para cada neurona oculta;

Calcular la salida para cada neurona de salida;

Acumular las diferencias entre las salidas actual y objetivo;

Calcular el gradiente modificado para $\Delta W_{k}$

Calcular el gradiente modificado para $\Delta W_{j}$

Modificar $\Delta W_{k j}$;

Modificar $\Delta W_{j i}$;

Fin

Fin

ENFOQUE

PROPUESTO

El enfoque propuesto para respuesta de calidad de manera cualitativa está dado por lo siguiente:

1. Establecer los conjuntos de entrenamiento y de prueba de la red uno. Tomar al azar $20 \%$ de los datos experimentales para formar el conjunto de prueba y los restantes forman el conjunto de entrenamiento.

2. Construir la arquitectura de la red uno y determinar el conjunto de parámetros óptimos para los factores controlables.

a. Calcular las probabilidades acumuladas para todas las respuestas cualitativas (en cada experimento, para cada nivel).

b. Construir la red uno, asignando la probabilidad acumulada de cada categoría de la respuesta cualitativa como el input; los correspondientes conjuntos de valores de los factores controlables son asignados como el output. Probar diversas arquitecturas de red bp mediante el uso del conjunto de entrenamiento y prueba seleccionados en el paso 1 (arquitectura: número de neuronas en la capa de entrada - capa oculta y capa de salida). El error cuadrado medio MSE de cada arquitectura puede ser utilizada como el criterio para determinar la mejor arquitectura. Un número predeterminado de ciclos de entrenamiento puede ser considerado como el criterio para detener el proceso de entrenamiento. La mejor arquitectura es aquella que puede simultáneamente minimizar el MSE del conjunto de entrenamiento y del de prueba del paso 1.

c. Ingrese las probabilidades acumuladas deseadas como input de la red uno encontrada en $b$ y calcule los output respectivos, que en este caso serán los factores controlables óptimos.
3. Construir la arquitectura de red dos para predecir las probabilidades acumuladas de las respuestas cualitativas.

a. Asignar el conjunto de valores de factores controlables de 1. como el input de la red dos. La correspondiente probabilidad acumulada de cada clase es asignada como la salida de la red dos.

b. Construir la red dos, probando varias arquitecturas de red dos (número de neuronas en las capas de entrada, oculta y de salida) mediante la combinación de los conjuntos de entrenamiento y prueba de 1 .

c. Ingrese el conjunto óptimo de parámetros encontrados en 2, para la mejor red backpropagation encontrada en b. La probabilidad acumulada predicha de cada categoría pueden ser entonces obtenida.

4. Comparar el resultado estimado con el objetivo. Si el resultado estimado en el paso 3.c no se aparta de manera significativa del valor objetivo, el análisis se ha completado. Si no fuera así, retorne al paso 1 para reformar el conjunto de entrenamiento y prueba y repita los pasos 2 a 4 hasta que la desviación entre los resultados estimados y el valor objetivo sea aceptable.

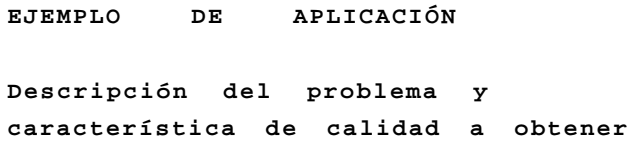
La característica de calidad que se buscaba es que no quede meloso a la semana de estar a la intemperie.

La melosidad es juzgada por inspección al tacto de los ingenieros de procesos. Mediante un número indicativo del nivel de adhesión de los dedos al estar en contacto con el caramelo es la forma de evaluación.

El nivel de melosidad puede ser clasificado en varias clases de acuerdo al nivel de adhesión que se produce al tacto. Los ingenieros de procesos determinaron 3 clases: la menor melosidad I, melosidad aceptable II, la peor melosidad - se derrite III.

El Cuadro 1 precisa la definición cualitativa de tres clases para la melosidad.

Reconocimiento del estado actual En planta los ingenieros escogen series pequeñas fuera de la línea para la inspección. Las pequeñas series fuera de la línea seleccionada son de 24 caramelos, los mismos que son evaluados mediante una inspección simple. 


\begin{tabular}{|c|c|}
\hline Categoría & Definición \\
\hline I. La menor melosidad & $\begin{array}{l}\text { Al tacto no queda nada pegajoso en el } \\
\text { dedo y no se aprecia variación sobre la } \\
\text { superficie } \\
\text { Al tacto los dedos quedan ligeramente } \\
\text { pegajosos, pero visualmente no se aprecia } \\
\text { variación sobre la superficie }\end{array}$ \\
\hline III La peor melosidad & $\begin{array}{l}\text { Al tacto los dedos quedan pegajosos, y } \\
\text { visualmente se aprecia variación sobre la } \\
\text { superficie }\end{array}$ \\
\hline
\end{tabular}

El estado actual de las probabilidades acumuladas, respectivamente, para las 3 clases I, II, III revela: $0.64000,0.7520,1.000$. Los resultados ideales de probabilidades acumuladas de las 3 clases son: $1.0000,1.0000,1.0000$. Obviamente, el resultado actual está lejos del resultado ideal. Para mejorar la característica de la melosidad, es necesario optimizar parámetros.

Determinación experimental de factores y diseño experimental

Hay varias variables del proceso que pueden afectar la calidad de la melosidad que se produce en la superficie después del proceso. Luego de analizar los mecanismos relacionados mediante un trabajo de tormenta de ideas, los ingenieros de procesos seleccionaron cuatro variables del proceso: la cantidad de azúcar que se agrega por lote, la temperatura de proceso, el tiempo de proceso y el tiempo de mezclado para obtener la masa caramelo.

El Cuadro 2 precisa los cuatro factores y sus definiciones de niveles. Para reducir el tiempo y costo experimental se selecciona un arreglo ortogonal L9, de los modelos de diseños experimentales que se proponen en los Métodos Taguchi (10). Cada prueba experimental se repitió 6 veces.

C ON C L U S I O N E S

En la actualidad en muchos casos dada la complejidad de los productos manufacturados, puede ser jus-
Cuadro 2. Definición de niveles de factores controlables

\begin{tabular}{|c|c|c|c|}
\hline Factor & Nivel 1 & Nivel 2 & Nivel 3 \\
\hline $\begin{array}{l}\text { A. Cantidad de azúcar que se } \\
\text { agrega por lote }(\mathrm{kg})\end{array}$ & $10^{*}$ & 12 & 14 \\
\hline B. Temperatura de proceso $\left({ }^{\circ} \mathrm{C}\right)$ & 75 & $80^{*}$ & 85 \\
\hline C. Tiempo de proceso (min) & 30 & 35 & $40^{*}$ \\
\hline $\begin{array}{l}\text { D. Tiempo de mezclado para } \\
\text { obtener la masa caramelo (min) } \\
\text { valor inicial estable cido para el factor cor }\end{array}$ & 20 & $25^{*}$ & 30 \\
\hline
\end{tabular}

tificado calcular una respuesta cuantitativa. La tendencia a emplear evaluaciones cualitativas, está aumentando gradualmente. Este estudio propone un nuevo enfoque basado en la técnica de redes neuronales para lograr los parámetros de optimización para respuestas de calidad con categorías de orden cualitativo. Después de obtener los parámetros de optimización necesarios, una mejora en la calidad puede ser lograda. El caso demuestra la efectividad del enfoque propuesto.

B I B I I O GR A F I A

1. April, J.; Glover, F.; Kelly, J. \& Laguna, M. (2003). Practical introduction to simulation optimization. Proceedings of the 2003 Winter Simulation Conference. OpTek Systems, USA.

2. Glover, F.; Kelly, J. \& Laguna, M. (1996). New advances and applications of combining simulation and optimization. Proceedings of the 28th Conference on Winter Simulation. ACM Press, New York, USA.

3. Hagan, M.; Howard, D. \& Beale, M. (1996). Neural Network Design. PWS-Kent Publishing Company, Boston, MA 02116, USA.

4. Hsieh, K.\& Tong, L. (1999). Parameteroptimization for quality response with linguistic ordered category by employing artificial neural networks: a case study. Journal of Science and Engineering. Vol. 4

Cuadro 3. Resultados de confirmación de experimentos para establecer los parámetros óptimos

\begin{tabular}{c|c|ccc|c|c|c|ccc}
\hline \multirow{2}{*}{$N^{\circ}$} & \multicolumn{4}{|c|}{ Parámetros establecidos } & \multicolumn{3}{c|}{$\begin{array}{c}\text { La cantidad de cada } \\
\text { clase }\end{array}$} & \multicolumn{3}{c}{$\begin{array}{c}\text { Las probabilidades } \\
\text { acumulada de cada clase }\end{array}$} \\
\cline { 2 - 10 } & $\mathbf{A}$ & B & C & D & I & II & III & [I] & [II] & [III] \\
1 & 10.2 & 79.0 & 36.1 & 19.6 & 22 & 2 & 0 & 0.9167 & 1.000 & 1.000 \\
2 & 10.2 & 79.0 & 36.1 & 19.6 & 21 & 2 & 1 & 0.8750 & 0.9583 & 1.000 \\
3 & 10.2 & 79.0 & 36.1 & 19.6 & 23 & 1 & 0 & 0.9583 & 1.0000 & 1.000 \\
\hline
\end{tabular}

[I], [II], [III] significan las probabilidades acumuladas de las clases I, II, III, respectivamente. 
Nro. 2. pp. 213-219. Tamkang University Press, Tamsui, Taiwan.

5. Hsieh, K. (2001). Process improvement in the presence of qualitative response by combining fuzzy sets and neural networks. Integrated Manufacturing Systems. Vol. 12, Nro.6, pp. 449-462.

6. Jeng, Y. C. \& Guo, S. M. (1996). Quality improvement for $R C 06$ chip resistor. Quality and Reliability Engineering International. Vol. 12. Issue 6. John Wiley \& Sons, Ltd. USA.

7. Konak, A.; Jonak, S.; Smith, A. \& Nettleship, J. (2001). Estimation of shrinkage for near-net shape using a neural network approach. Journal of Intelligent Manufacturing, Special Issue on Soft Computing in Manufacturing, December 2001.

8. Martin Del Brio, B y Sanz, A. (2002). Redes neuronales y Sistemas Difusos. Editorial Alfaomega. Madrid, España.

9. Nair, V.N. (1986). Testing in Industrial Experiments with ordered categorical data. Technometrics. Vol 28, pp. 283-291.

10.Taguchi, G.; Chowdhury, S. \& Wu, Y. (2004) Taguchi's Quality Engineering Handbook. WileyInterscience. USA. 